\title{
Exact eigenstates and macroscopic magnetization jumps in strongly frustrated spin lattices
}

\author{
J. Richter $§$, J. Schulenburg $\ddagger$, A. Honecker§, J. Schnack $\|$ and \\ H.-J. Schmidt $\|$ \\ $\dagger$ Institut für Theoretische Physik, Universität Magdeburg, P.O. Box 4120, D-39016 \\ Magdeburg, Germany \\ $\ddagger$ Universitätsrechenzentrum, Universität Magdeburg \\ §Institut für Theoretische Physik, TU Braunschweig \\ || Universität Osnabrück, Fachbereich Physik
}

\begin{abstract}
.
For a class of frustrated spin lattices including e.g. the 1D sawtooth chain, the 2D kagomé and checkerboard, as well as the 3D pyrochlore lattices we construct exact product eigenstates consisting of several independent, localized one-magnon states in a ferromagnetic background. Important geometrical elements of the relevant lattices are triangles being attached to polygons or lines. Then the magnons can be trapped on these polygons/lines. If the concentration of localized magnons is small they can be distributed randomly over the lattice. Increasing the number of localized magnons their distribution over the lattice becomes more and more regular and finally the magnons condensate in a crystal-like state.

The physical relevance of these eigenstates emerges in high magnetic fields where they become groundstates of the system. As a result a macroscopic magnetization jump appears in the zero-temperature magnetization curve just below the saturation field. The height of the jump decreases with increasing spin quantum number and vanishes in the classical limit. Thus it is a true macroscopic quantum effect.
\end{abstract}

submitted to: J. Phys.: Condens. Matter

PACS numbers: 75.10.Jm, 75.45.+j, 75.50.Ee, 75.60.Ej

\section{Introduction}

The search for exact eigenstates of quantum spin systems attracts continuous attention ever since the Heisenberg and related spin models are studied. Of course we have the fully polarized ferromagnetic state as a trivial example. Furthermore the one- and twomagnon states above the fully polarized ferromagnetic state can be calculated exactly (see, e.g. [1]). A famous example for a very non-trivial eigenstate is Bethe's solution for the groundstate of the one-dimensional (1D) Heisenberg antiferromagnet (HAFM) [2].

The investigation of strongly frustrated magnetic systems surprisingly led to the discovery of several new exact eigenstates. Whereas in general the treatment of $\S$ To whom correspondence should be addressed (johannes.richter@physik.uni-magdeburg.de) 
frustrated quantum magnets is even harder than that of unfrustrated systems in some exceptional cases one finds eigenstates of quite simple nature. The interest in these eigenstates comes from the fact that often they become groundstates either for particular values of frustration or in magnetic fields. Furthermore the spin correlation functions can be calculated analytically. Therefore these exact eigenstates play an important role either as groundstates of real quantum magnets or at least as reference states of idealized models for more complex quantum spin systems. There are two well-known examples for simple eigenstates of strongly frustrated quantum spin systems, namely the MajumdarGosh state of the 1D $J_{1}-J_{2}$ spin 1/2 HAFM [3] and the orthogonal dimer state in the Shastry-Sutherland model [4]. Both eigenstates are products of dimer singlets and become groundstates only for strong frustration. These eigenstates indeed play a role in realistic materials. While the Majumdar-Ghosh state has some relevance in quasi$1 \mathrm{D}$ spin-Peierls materials like $\mathrm{CuGeO}_{3}$ (see e.g. [5]), the orthogonal dimer state of the Shastry-Sutherland model is the magnetic groundstate of the quasi-2d $\mathrm{SrCu}_{2}\left(\mathrm{BO}_{3}\right)_{2}[6$. Other frustrated spin models in one, two or three dimensions can be constructed having also dimer singlet product states as groundstates (see e.g. [7, 8, 9]).

Less known is the so-called uniformly distributed resonating valence bond state which is the groundstate of the $J_{1}-J_{2}$ chain with ferromagnetic nearest-neighbour $(\mathrm{NN})$ bonds $J_{1}<0$ and frustrating antiferromagnetic next $\mathrm{NN}$ bonds $J_{2}=-J_{1} / 4[10]$.

Another striking feature of the dimer singlet product groundstates is the existence of magnetization plateaus at zero magnetization. These plateaus in quantum spin systems currently attract a lot of attention from the theoretical as well as experimental side. Recently it has been demonstrated for the 1d counterpart of the Shastry-Sutherland model, the so-called frustrated dimer-plaquette chain (also known as orthogonal dimer chain) [8, 11, 12, that more general product eigenstates containing chain fragments of finite length lead to an infinite series of magnetization plateaus [13].

In this paper we discuss a recently discovered [14] class of quite universal eigenstates of frustrated quantum antiferromagnets which become groundstates in strong magnetic fields and lead to macroscopic jumps in the magnetization curve close to saturation. In what follows we consider in more detail the sawtooth chain and the checkerboard lattice.

\section{Localized magnon states}

We consider $N$ quantum spins of "length" $s$ described by the Heisenberg Hamiltonian

$$
\hat{H}=\sum_{i j} J_{i j}\left\{s_{i}^{x} s_{j}^{x}+s_{i}^{y} s_{j}^{y}+s_{i}^{z} s_{j}^{z}\right\}-h S^{z} .
$$

$S^{z}=\sum_{i} s_{i}^{z}$ is the $z$-component of the total spin, $h$ is the magnetic field, and the $J_{i j}$ are the exchange constants.

If the magnetic field $h$ is sufficiently strong $h \geq h_{s}$, the groundstate of (11) becomes

the fully polarized ferromagnetic state (magnon vacuum state) $|0\rangle=|\uparrow \uparrow \uparrow \ldots\rangle$ where all spins assume their maximal $s_{i}^{z}$-quantum number and $M=\left\langle S_{z}\right\rangle=N s$. The lowest excitations for $h>h_{s}$ are one-magnon states $|1\rangle$ with $M=N s-1$. They can be 
written as $|1\rangle \sim \frac{1}{c} \sum_{i}^{N} a_{i} s_{i}^{-}|0\rangle$, where in general the excitation is distributed over the whole system. However, for highly frustrated magnets having special bond geometry it turns out that the excitation can be localized over a restricted area $L$ of the system, i.e. we have

$$
|1\rangle \rightarrow|1\rangle_{L}=\frac{1}{c} \sum_{i \in L} a_{i} s_{i}^{-}|0\rangle=\left|\Psi_{L}\right\rangle\left|\Psi_{R}\right\rangle,
$$

where $\left|\Psi_{L}\right\rangle$ is the wave function of the magnon localized on area $L$ and $\left|\Psi_{R}\right\rangle$ the wave function of the fully polarized ferromagnetic remainder $R$ containing all sites not belonging to $L$. In (2) the constant $c$ is chosen to normalize $|1\rangle_{L}$. To demonstrate this we split the Hamiltonian into three parts $\hat{H}=\hat{H}_{L}+\hat{H}_{L-R}+\hat{H}_{R}$, where $\hat{H}_{L}\left(\hat{H}_{R}\right)$ contains only spins belonging to the local area $L$ (remainder $R$ ) and $\hat{H}_{L-R}$ is the interaction term between $L$ and $R$. We restrict our consideration to the case that the wave function $\left|\Psi_{L}\right\rangle$ is an eigenstate of $\hat{H}_{L}$ and has lowest one-magnon energy. Of course, the wave function $\left|\Psi_{R}\right\rangle$ is an eigenstate of $\hat{H}_{R}$. Now we demand that the total wave function (2) is an eigenstate of the full Hamiltonian $\hat{H}_{L}+\hat{H}_{L-R}+\hat{H}_{R}$. After some manipulation one finds that the exchange couplings in the interaction term $\hat{H}_{L-R}$ have to fulfill two conditions [15], namely

$$
\sum_{l \in L} J_{r l} a_{l}=0 \quad \forall r \in R
$$

and

$$
\sum_{r \in R} J_{r l}=\text { const. } \quad \forall l \in L
$$

The first one comes from the xx and yy terms in $\hat{H}_{L-R}$ and the second one from the zz term (i.e. condition (4) is not relevant for the pure xy model). Eq. (30) leads to condition on the bond geometry, whereas eq. (44) is a condition for the bond strengths and is automatically fulfilled in uniform lattices with equivalent sites. Indeed one finds that the above conditions are fulfilled for remarkably many different lattices/models such as the kagomé and the sawtooth chain, the 2 d kagomé, square-kagomé or checkerboard lattices, the $3 \mathrm{~d}$ pyrochlore lattice but also for a fractal lattice like the Sierpinski gasket.

In Fig.1 we illustrate the localized magnon states on the sawtooth chain with $J_{2}=2 J_{1}$ and on the checkerboard lattices. Both systems attract currently a lot of attention as examples for novel low-energy physics in quantum systems (see e.g. [16, 17]). We show in the figures only the localized magnons of minimum size. On the checkerboard lattice localized magnons can sit on the four sites of an 'empty' square but also on the $\sqrt{N}$ sites of a vertical, horizontal or sloping $\left(45^{\circ}\right)$ line. On the sawtooth chain a magnon can sit on the three neighbouring sites forming ' $\mathbf{V}$ ' but also on the $N / 2$ sites on the base line.

The next step is to construct eigenstates containing $n>1$ localized magnons on different localized areas $L_{\alpha}, \alpha=1, \ldots, n$. If these localized areas are separated from each other one can still fulfill both conditions (3) and (4). This way one can find a whole class of product eigenstates of the form $|\Psi\rangle=\left|\Psi_{L_{1}}\right\rangle\left|\Psi_{L_{2}}\right\rangle \cdots\left|\Psi_{L_{m}}\right\rangle\left|\Psi_{R}\right\rangle$, where 

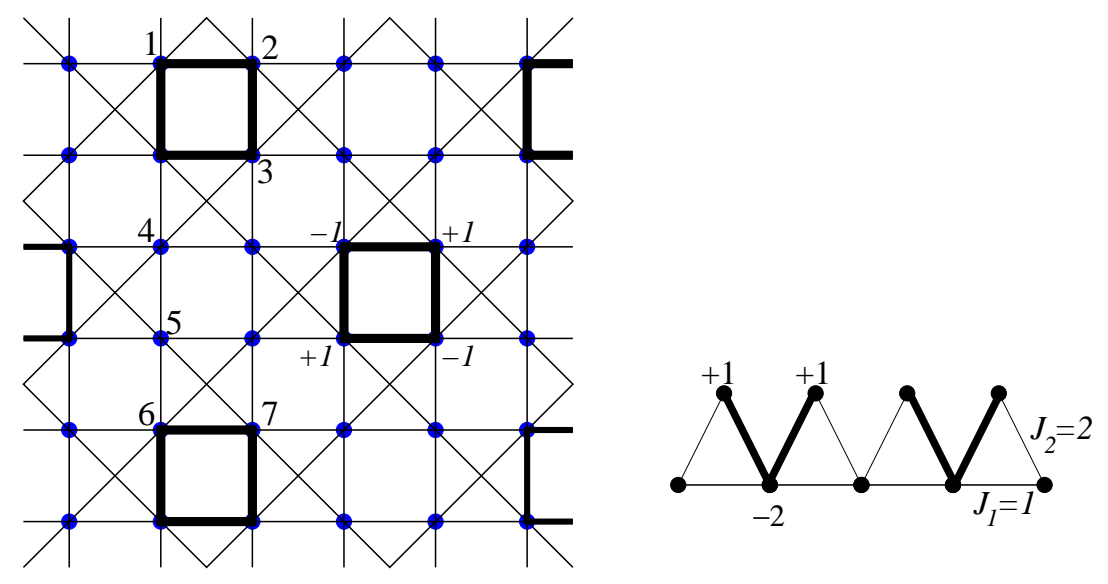

Figure 1. Localized magnon states on the checkerboard lattice and on the sawtooth chain with $J_{2}=2 J_{1}$. The position of the localized magnons are indicated by extra thick lines. The numbers $+1,-1,-2$ at the corners represent the coefficients $a_{i}$ (see eq. (21)).

the $\left|\Psi_{L_{\alpha}}\right\rangle$ and $\left|\Psi_{R}\right\rangle$ are defined in analogy to (2). The system can be filled with separated magnons as long as the magnons do not interact. The maximum number of localized magnons depends on the lattice geometry. The state with maximum filling corresponds to a 'magnon crystal', i.e. to a state with a regular arrangement of magnons (see Fig (1). The 'crystalline' magnon state of the sawtooth chain (checkerboard lattice) is twofold (fourfold) degenerated and breaks spontaneously the translational symmetry of the lattice. Maximum filling for the checkerboard lattice is reached if magnons sit on every forth 'empty' square and for the sawtooth chain if magnons sit on every second 'V'. Each localized magnon lowers the magnetic quantum number $M$ of total $S_{z}$ by one, i.e. one has $M=N s-n$ for a state with $n$ localized magnons. Hence the corresponding quantum number of the 'magnon crystal' is $M=N s-N / 8$ (checkerboard) and $M=N s-N / 4$ (sawtooth).

Due to the simple product form of the eigenstates one can calculate explicitely the spin-spin correlation functions. They depend on the parameters $a_{i}$. One has three different types of correlations, namly within $R$, within $L$ and between $L$ nad $R$. As an example we give the correlations for the checkerboard lattice with localized magnons on 'empty' squares: $\left\langle\vec{s}_{1} \vec{s}_{2}\right\rangle=-1 / 4 ;\left\langle s_{1}^{z} s_{2}^{z}\right\rangle=0 ;\left\langle\vec{s}_{1} \vec{s}_{3}\right\rangle=1 / 4 ;\left\langle s_{1}^{z} s_{3}^{z}\right\rangle=0 ;\left\langle\vec{s}_{1} \vec{s}_{4}\right\rangle=\left\langle s_{1}^{z} s_{4}^{z}\right\rangle=$ $1 / 8 ;\left\langle\vec{s}_{1} \vec{s}_{6}\right\rangle=\left\langle s_{1}^{z} s_{6}^{z}\right\rangle=\left\langle\vec{s}_{1} \vec{s}_{7}\right\rangle=\left\langle s_{1}^{z} s_{7}^{z}\right\rangle=1 / 16$ and $\left\langle\vec{s}_{4} \vec{s}_{5}\right\rangle=\left\langle s_{4}^{z} s_{5}^{z}\right\rangle=1 / 4$ (the numbers correspond to those given in Fig. 11). Other correlation functions can be obtained by symmetry arguments having in mind that there is no real distance dependence of correlations within $R$ and between $L$ and $R$.

Finally, we mention that the existence of localized magnon states in regular spin lattices is related to the existence of flat bands in the magnon dispersion[14]. 

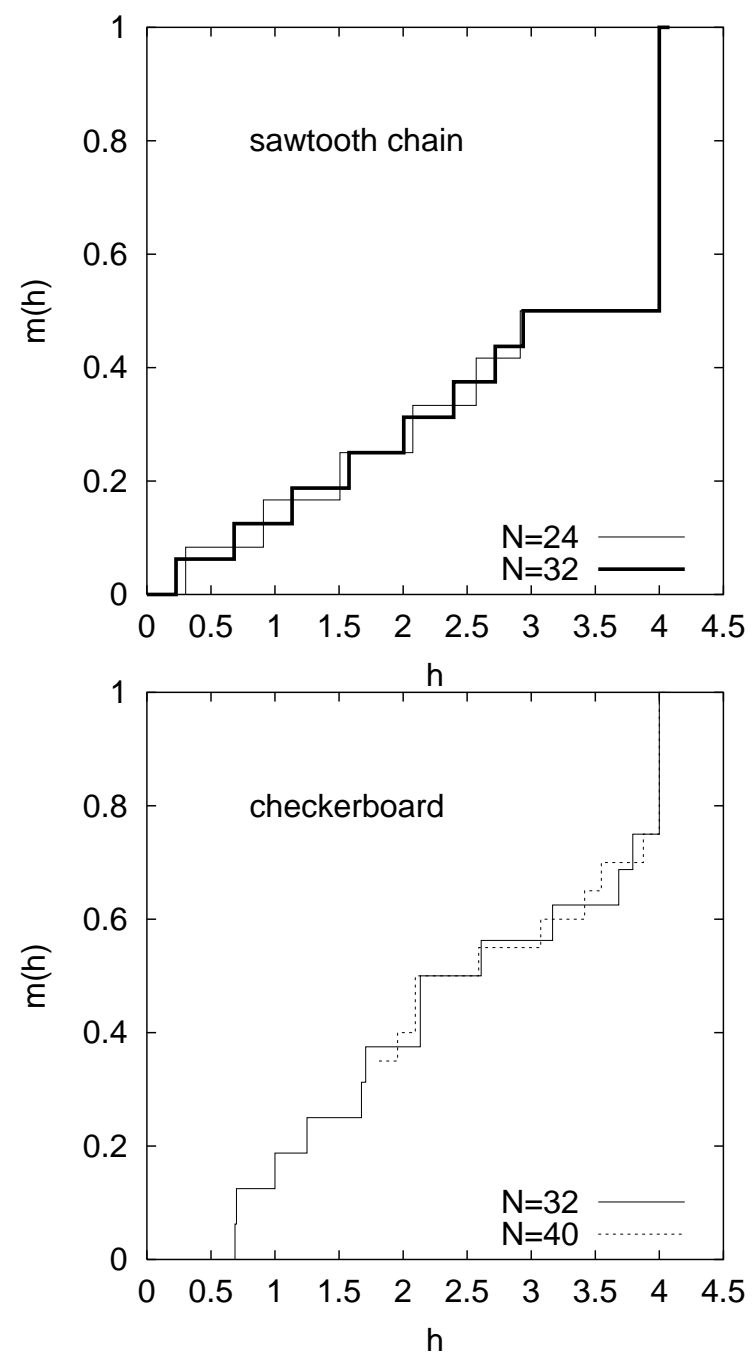

Figure 2. Magnetization $m$ versus magnetic field $h$ of the spin half Heisenberg antiferromagnet on the sawtooth chain $\left(J_{2}=2 J_{1}\right.$, cf. Fig. 1) and on the checkerboard lattice. For the finite checkerboard lattice of $N=40$ only the upper part of the curve was calculated.

\section{Macroscopic magnetization jump}

The interest in the above described eigenstates is not only an academic one. One can show rigorously that these eigenstates under certain conditions become groundstates in a magnetic field [18]. This is true for instance in many systems with translational symmetry. We define as magnetization $m=\frac{\left\langle S^{z}\right\rangle}{N s}=\frac{M}{M_{0}}$, i.e. $m$ is normalized to unity for the fully polarized ferromagnetic state $|0\rangle$. Since the $\mathrm{z}$ component of the total spin commutes with the Hamiltonian one can calculate the magnetization from the lowest zero-field energies $E(M)$ in each sector of $M$, i.e. $E(M, h)=E(M)-h M$ and $h(M)=\frac{\Delta E}{\Delta M}$. The eigenstates discussed in section 2 contain localized noninteracting magnons and have therefore well defined energies $E(M=N s-n)=N s^{2}-n \varepsilon_{1}$, where 
$\varepsilon_{1}$ is the energy difference between the magnon vacuum and state with one magnon and $n$ is the number of localized magnons. Hence we have a straight part in the $E$ versus $M$ curve close to saturation leading to a jump in the $m(h)$ curve. The height of the jump $\Delta m$ is determined by the maximal number $n_{\max }$ of localized magnons in the system and on the spin 'length' $s, \Delta m=n_{\max } / N s$. Obviously the jump is a true quantum effect and vanishes for $s \rightarrow \infty$.

In Fig. 2]we show magnetization curves for the sawtooth chain and the checkerboard lattice for $s=1 / 2$ calculated with exact diagonalization for finite lattices. The height of the jump for the sawtooth chain is $\Delta m=1 / 2$ and for the checkerboard lattice $\Delta m=1 / 4$. We emphasize that $\Delta m$ does not depend on the size of the system provided the periodic boundary conditions of the finite lattice fit to the translational symmetry of the 'magnon crystal'. For the sawtooth chain we see a well-pronounced plateau preceding the jump. This plateau belongs to the 'magnon crystal' state. Though such a plateau is less pronounced for the checkerboard lattice there are general arguments 19] that such a crystalline magnon state should have gapped excitations and may therefore be connected with a magnetization plateau at $m=1-\delta m=1-n_{\max } / N s$. We mention that the plateaus fulfill the condition of Oshikawa, Yamanaka and Affleck [20] derived for plateaus in $1 \mathrm{~d}$ systems not only for the sawtooth chain but also for the $2 \mathrm{~d}$ checkerboard lattice.

To our knowlegde the jump was not observed experimentally so far. To see the jump and the preceding plateau in experiments one needs highly frustrated magnets with small spin quantum number $s$ and sufficiently small exchange coupling strength $J$ to reach the saturation field.

\section{Acknowledgments}

This work was supported by the Deutsche Forschungsgemeinschaft (Grant No. Ri615/10-1).

\section{References}

[1] Mattis D C The Theory of Magnetism I, Springer, Berlin 1988

[2] Bethe H A 1931 Z. Phys. 71205

[3] Majumdar C K and Ghosh D K 1969 J. Math. Phys. 101399

[4] Shastry B S and Sutherland B 1981 Physica 108B 1069

[5] Poilblanc D, Riera J, Hayward C A, Berthier C and Horvatic M 1997 Phys. Rev. B 55941

[6] Miyahara S and Ueda K 1999 Phys. Rev. Lett. 82, 3701

[7] Pimpinelli A 1991 J. Phys.: Condens. Matter 3445

[8] Ivanov N B and Richter J 1997 Phys. Lett. A 232308 ; Richter J, Ivanov N B and Schulenburg J 1998 J. Phys.: Condens. Matter 103635

[9] Ueda K and Miyahara S 1999 J. Phys.: Condens. Matter 11 L175

[10] Hamada T, Kane J, Nagawaka S and Natsume Y J. Phys. Soc. Japan 1988571891

[11] Koga A, Okunishi K, and Kawakami N 2000 Phys. Rev. B 625558 ; Koga A and Kawakami N 2002 Phys. Rev. B 65, 214415 
[12] Schulenburg J and Richter J 2002 Phys. Rev. B 66134419

[13] Schulenburg J and Richter J 2002 Phys. Rev. B 65054420

[14] Schulenburg J, Honecker A, Schnack J, Richter J and Schmidt H-J 2002 Phys. Rev. Lett. 88167207

[15] The second condition is not a necessary one, i.e. one can find models with eigenstates of form (2) violating (4), see [14]. This more general case appears if $\left|\Psi_{L}\right\rangle\left|\Psi_{R}\right\rangle$ is not an individual eigenstate of both $\hat{H}_{L}$ and $\hat{H}_{L-R}$ but of $\left(\hat{H}_{L-R}+\hat{H}_{L}\right)$.

[16] Palmer S E and Chalker J T 2001 Phys. Rev. B 64, 094412 ; Brenig W and Honecker A 2002 Phys. Rev. B 65 R140407 ; Fouet J B, Mambrini M, Sindzingre P and Lhuillier C 2003 Phys. Rev. B 67054411

[17] Sen D, Shastry B S, Walstedt R E, Cava R 1996 Phys. Rev. B 536401 ; Pati S K 2003 Phys. Rev. B 67184411 ; Chandra V R, Sen D, Ivanov N B and Richter J 2003 cond-mat/0307492

[18] Schnack J, Schmidt H-J, Richter J and Schulenburg J 2002 Eur. Phys. J. B 200124475 ; Schmidt H-J 2002 J. Phys. A: Math. Gen. 356545

[19] Momoi T and Totsuka K 2000 Phys. Rev. B 613231 ; Oshikawa M 2000 Phys. Rev. Lett. 841535

[20] Oshikawa M, Yamanaka M and Affleck I 1997 Phys. Rev. Lett. 781984 